\title{
WEAK CONVERGENCE OF FINITE ELEMENT APPROXIMATIONS OF LINEAR STOCHASTIC EVOLUTION EQUATIONS WITH ADDITIVE NOISE
}

\author{
MIHÁLY KOVÁCS, STIG LARSSON ${ }^{1,2}$, AND FREDRIK LINDGREN ${ }^{1}$
}

\begin{abstract}
A unified approach is given for the analysis of the weak error of spatially semidiscrete finite element methods for linear stochastic partial differential equations driven by additive noise. An error representation formula is found in an abstract setting based on the semigroup formulation of stochastic evolution equations. This is then applied to the stochastic heat, linearized Cahn-Hilliard, and wave equations. In all cases it is found that the rate of weak convergence is twice the rate of strong convergence, sometimes up to a logarithmic factor, under the same or, essentially the same, regularity requirements.
\end{abstract}

\section{INTRODUCTION}

Let $U, H$ be real separable Hilbert spaces and consider the following abstract stochastic Cauchy problem

$$
\mathrm{d} X(t)+A X(t) \mathrm{d} t=B \mathrm{~d} W(t), t>0 ; \quad X(0)=X_{0},
$$

where $-A$ is the generator of a strongly continuous semigroup $\{E(t)\}_{t>0}$ on $H$, $B \in \mathcal{B}(U, H)$, where $\mathcal{B}(U, H)$ denotes the space of bounded linear operators from $U$ to $H,\{W(t)\}_{t \geq 0}$ is a $U$-valued Wiener process with covariance operator $Q$ with respect to a filtration $\left\{\mathcal{F}_{t}\right\}_{t \geq 0}$ on a probability space $(\Omega, \mathcal{F}, P)$, and $X_{0}$ is an $\mathcal{F}_{0^{-}}$ measurable $H$-valued random variable. The covariance operator $Q \in \mathcal{B}(U, U)$ with $Q \geq 0$ (selfadjoint, positive semidefinite). Under appropriate conditions, see 3.5 below, the unique weak solution is given by

$$
X(t)=E(t) X_{0}+\int_{0}^{t} E(t-s) B \mathrm{~d} W(s) .
$$

Let $V_{h} \subset H$ be a family finite dimensional subspaces and let $B_{h} \in \mathcal{B}(U, H)$ be a family of operators with $B_{h}: U \rightarrow V_{h}, 0<h \leq 1$. We consider approximating stochastic Cauchy problems of the form

$$
\mathrm{d} X_{h}(t)+A_{h} X_{h}(t) \mathrm{d} t=B_{h} \mathrm{~d} W(t), t>0 ; \quad X_{h}(0)=X_{h 0},
$$

Date: September 6, 2010.

2000 Mathematics Subject Classification. 65M60, 60H15, 60H35, 65C30.

Key words and phrases. finite element, parabolic equation, hyperbolic equation, stochastic, heat equation, Cahn-Hilliard-Cook equation, wave equation, additive noise, Wiener process, error estimate, weak convergence.

${ }^{1}$ Supported by the Swedish Research Council (VR).

${ }^{2}$ Supported by the Swedish Foundation for Strategic Research (SSF) through GMMC, the Gothenburg Mathematical Modelling Centre. 
where $-A_{h}$ is the generator of a strongly continuous semigroup $\left\{E_{h}(t)\right\}_{t \geq 0}$ on $V_{h}$. We take $X_{h 0}$ to be an $\mathcal{F}_{0}$-measurable random variable. As above the unique weak solution is given by

$$
X_{h}(t)=E_{h}(t) X_{h 0}+\int_{0}^{t} E_{h}(t-s) B_{h} \mathrm{~d} W(s) .
$$

This framework is designed to accomodate standard spatial finite element discretizations of various linear stochastic evolution problems including the heat equation, the linearized Cahn-Hilliard equation, and the wave equation. For further details on stochastic integration and the semigroup approach to stochastic partial differential equations we refer to 1 .

Let $G: H \rightarrow \mathbb{R}$ be a function with globally bounded, continuous Fréchet derivatives of order 1 and 2 , that is, $G \in C_{\mathrm{b}}^{2}(H, \mathbb{R})$. We consider the weak error $e_{h}(T)$ at $T>0$ defined as

$$
e_{h}(T):=\mathbf{E}\left(G\left(X_{h}(T)\right)\right)-\mathbf{E}(G(X(T))) .
$$

While the literature on strong convergence of numerical approximations of stochastic partial differential equations is abundant, especially for parabolic problems (see [5] for an exhaustive list of references), there is very little on weak convergence. In particular, there are no results on the weak error of the finite element method for the linear stochastic Cahn-Hilliard and wave equations. The papers [4, 5, 9] consider the stochastic heat equation and so does [7], which proves similar results but under a stronger restriction on the test function $G$. The results in [3] are concerned with the Schrödinger equation and [8] proves weak convergence of the leap frog scheme for the stochastic wave equation. In all cases it is observed that the rate of weak convergence is twice that of strong convergence.

We now present a brief outline of this paper. Precise definitions and statements are given in the following sections. In Section 2 we recall basic facts about trace class and Hilbert-Schmidt operators. In Section 3 we work in the abstract setting (1.2), (1.4) and derive a formula for the weak error in Theorem 3.1. This is then applied to semidiscretizations of parabolic equations in Section 4 and a hyperbolic equation in Section 5. An important difference is that the semigroup $E(t)=\mathrm{e}^{-t A}$ is analytic in Section 4 but only strongly continuous in Section 5 .

Let $\mathcal{D} \subset \mathbb{R}^{d}$ be a spatial domain and consider the Laplace operator $\Lambda=-\Delta$ as an unbounded operator on $L_{2}(\mathcal{D})$ with domain of definition $D(\Lambda)=H^{2}(\mathcal{D}) \cap H_{0}^{1}(\mathcal{D})$. In Subsection 4.1 we study the stochastic heat equation,

$$
\mathrm{d} X+\Lambda X \mathrm{~d} t=\mathrm{d} W, t>0 ; \quad X(0)=X_{0} .
$$

This is of the form (1.1) with $H=U=L_{2}(\mathcal{D}), A=\Lambda, B=I$.

Let $S_{h} \subset H_{0}^{1}(\mathcal{D})$ be a family of standard finite element spaces consisting of continuous piecewise polynomials of degree $\leq r-1$ parametrized by meshsize $h$. Thus $r \geq 2$ is the formal convergence order of the finite element method. The spatially discrete approximation of $(1.6)$ is

$$
\mathrm{d} X_{h}+\Lambda_{h} X_{h} \mathrm{~d} t=P_{h} \mathrm{~d} W, t>0 ; \quad X_{h}(0)=P_{h} X_{0}
$$

Here $\Lambda_{h}$ denotes the discrete Laplacian and $P_{h}: L_{2}(\mathcal{D}) \rightarrow S_{h}$ is the orthogonal projection. This is clearly of the form 1.3 with $V_{h}=S_{h}, A_{h}=\Lambda_{h}, B_{h}=P_{h}$, $X_{h 0}=P_{h} X_{0}$. 
In 14 it was assumed that

$$
\left\|\Lambda^{\frac{\beta-1}{2}} Q^{\frac{1}{2}}\right\|_{\mathrm{HS}}<\infty, \quad \text { for some } \beta \geq 0,
$$

where $\|\cdot\|_{\text {HS }}$ denotes the Hilbert-Schmidt norm of bounded linear operators, see (2.1). Under appropriate smoothness of the initial value it was then shown that the solution has regularity of order $\beta$ in the mean square,

$$
\left(\mathbf{E}\left(\left\|\Lambda^{\frac{\beta}{2}} X(t)\right\|^{2}\right)\right)^{1 / 2}<\infty
$$

and that the finite element approximation has strong convergence of order $\beta$,

$$
\left(\mathbf{E}\left(\left\|X_{h}(t)-X(t)\right\|\right)^{2}\right)^{1 / 2}=O\left(h^{\beta}\right), \quad 0 \leq \beta \leq r .
$$

Here $\|\cdot\|$ denotes the norm in $H=L_{2}(\mathcal{D})$.

In the present work we first show in Theorem 4.1 that under the condition (1.8) we have weak convergence of essentially order $2 \beta$,

$$
e_{h}(T)=O\left(h^{2 \beta}|\log (h)|\right), \quad 0<\beta \leq 1 .
$$

For larger $\beta$ we assume in Theorem 4.2 that

$$
\left\|\Lambda^{\beta-1} Q\right\|_{\operatorname{Tr}}<\infty,
$$

where the trace norm $(2.3)$ is used, and we show weak order $O\left(h^{2 \beta}|\log (h)|\right)$ for $1 \leq \beta \leq \frac{r}{2}$.

In order to compare 1.12 and 1.8 , we show in Theorem 2.1 that

$$
\left\|\Lambda^{\frac{\beta-1}{2}} Q^{\frac{1}{2}}\right\|_{\mathrm{HS}} \leq\left\|\Lambda^{\beta-1} Q\right\|_{\mathrm{Tr}} \leq\left\|\Lambda^{\beta-1+\alpha} Q\right\|_{\mathcal{B}(H)}\left\|\Lambda^{-\alpha}\right\|_{\operatorname{Tr}}, \quad \beta \geq 0, \alpha>0 .
$$

It is clear that 1.12 implies 1.8 and that they coincide in two important cases: (i) if $\Lambda$ and $Q$ commute, in particular, if $Q=I$; and (ii) if $\beta=1$, that is, if $\operatorname{Tr}(Q)<\infty$. Thus, the rate of weak convergence is essentially twice the rate of strong convergence under essentially the same regularity assumption.

A result similar to (1.11) was first proved in [5. More precisely, there it was assumed that

$$
\left\|\Lambda^{\delta} Q\right\|_{\mathcal{B}(H)}<\infty,\left\|\Lambda^{-\alpha}\right\|_{\operatorname{Tr}}<\infty, \quad \text { for some } \alpha>0, \alpha-1 \leq \delta \leq \alpha .
$$

In view of 1.13 is is clear that (1.14) implies 1.8 with $\beta=1-\alpha+\delta$. Under this assumption was shown in [5] that we have weak convergence of order $O\left(h^{2 \gamma}\right)$ for $0<\gamma<\beta \leq 1$, which is almost 1.11). Weak convergence of the form (1.11) was also proved in [9] under assumption (1.8) but with stronger restrictions on the test function $G$ and on $r$.

Hence, for the stochastic heat equation, we slightly sharpen and simplify the results of [5] and 9] and we extend them to higher order.

In Subsection 4.2 study the linearized stochastic Cahn-Hilliard equation (linearized Cahn-Hilliard-Cook equation),

$$
\mathrm{d} X+\Lambda^{2} X \mathrm{~d} t=\mathrm{d} W, t>0 ; \quad X(0)=X_{0},
$$

with finite element approximation

$$
\mathrm{d} X_{h}+\Lambda_{h}^{2} X_{h} \mathrm{~d} t=P_{h} \mathrm{~d} W, t>0 ; \quad X_{h}(0)=P_{h} X_{0} .
$$

Under the assumption $\left\|\Lambda^{\frac{\beta-2}{2}} Q^{\frac{1}{2}}\right\|_{\mathrm{HS}}<\infty$, it was shown in [11 that we have regularity of order $\beta$ for $\beta \geq 0$ and strong convergence of order $O\left(h^{\beta}|\log (h)|\right)$ for $1 \leq \beta \leq r$. Here, in Theorem 4.4, if we assume, for example, that $S_{h}$ is based on 
a quasi-uniform mesh family and that for some $\alpha>0$ we have $0<\beta \leq \min \left(2, \frac{r}{2}\right)$, $0 \leq \beta-2+\alpha \leq 1$ and

$$
\left\|\Lambda^{\beta-2+\alpha} Q\right\|_{\mathcal{B}(H)}<\infty, \quad\left\|\Lambda^{-\alpha}\right\|_{\operatorname{Tr}}<\infty,
$$

then the weak convergence is of order $O\left(h^{2 \beta}|\log (h)|\right)$.

Our most novel result concerns the stochastic wave equation in Section 5 ,

$$
\begin{array}{ll}
\mathrm{d} X_{1}-X_{2} \mathrm{~d} t=0, t>0 ; & X_{1}(0)=X_{0,1}, \\
\mathrm{~d} X_{2}+\Lambda X_{1} \mathrm{~d} t=\mathrm{d} W, t>0 ; & X_{2}(0)=X_{0,2},
\end{array}
$$

with its straight-forward finite element approximation based on $S_{h}$ and $\Lambda_{h}$. This is of the form (1.1) with $H=L_{2}(\mathcal{D}) \times\left(H_{0}^{1}(\mathcal{D})\right)^{*}, U=L_{2}(\mathcal{D})$, and

$$
A=\left[\begin{array}{cc}
0 & -I \\
\Lambda & 0
\end{array}\right], \quad B=\left[\begin{array}{l}
0 \\
I
\end{array}\right], \quad X=\left[\begin{array}{l}
X_{1} \\
X_{2}
\end{array}\right], \quad X_{0}=\left[\begin{array}{c}
X_{0,1} \\
X_{0,2}
\end{array}\right] .
$$

Under assumption (1.8) it was shown in [10] for the first component $X_{1}$ (the displacement) that we have regularity of order $\beta$ for $\beta \geq 0$ and strong convergence of order $O\left(h^{\frac{r}{r+1} \beta}\right)$ for $0 \leq \beta \leq r+1$. Here, in Theorem 5.1. we assume

$$
\left\|\Lambda^{\beta-\frac{1}{2}} Q \Lambda^{-\frac{1}{2}}\right\|_{\operatorname{Tr}}<\infty
$$

and show weak convergence of order $O\left(h^{\frac{r}{r+1} 2 \beta}\right)$ for $0 \leq \beta \leq \frac{r+1}{2}$. Again, we show in Theorem 2.1 that the new condition (1.18) implies (1.8) and that they coincide if $\Lambda$ and $Q$ commute.

\section{Preliminaries}

Let $H$ be a separable real Hilbert space with scalar product $\langle\cdot, \cdot\rangle$ and norm $\|\cdot\|$ and let $\mathcal{B}(H)$ denote the space of bounded linear operators on $H$ with the usual norm $\|\cdot\|_{\mathcal{B}(H)}$. An operator $T \in \mathcal{B}(H)$ is called Hilbert-Schmidt, if for some orthonormal basis $\left\{e_{k}\right\}_{k=1}^{\infty}$ the sum

$$
\|T\|_{\mathrm{HS}}^{2}:=\sum_{k=1}^{\infty}\left\|T e_{k}\right\|^{2}
$$

is finite. In this case the sum is independent of the choice of the orthonormal basis and the quantity $\|T\|_{\mathrm{HS}}$ is called the Hilbert-Schmidt norm of $T$. The set of Hilbert-Schmidt operators is denoted by $\mathcal{L}_{2}(H)$. If $S \in \mathcal{B}(H)$ and $T \in \mathcal{L}_{2}(H)$, then $T^{*}, T S$, and $S T$ belong to $\mathcal{L}_{2}(H)$ and

$$
\left\|T^{*}\right\|_{\mathrm{HS}}=\|T\|_{\mathrm{HS}},\|T S\|_{\mathrm{HS}} \leq\|T\|_{\mathrm{HS}}\|S\|_{\mathcal{B}(H)},\|S T\|_{\mathrm{HS}} \leq\|T\|_{\mathrm{HS}}\|S\|_{\mathcal{B}(H)} .
$$

Let $\mathcal{L}_{1}(H)$ denote the set of nuclear operators from $H$ to $H$, that is, $T \in \mathcal{L}_{1}(H)$ if $T \in \mathcal{B}(H)$ and there are sequences $\left\{a_{j}\right\},\left\{b_{j}\right\} \subset H$ with $\sum_{j=1}^{\infty}\left\|a_{j}\right\|\left\|b_{j}\right\|<\infty$ and such that

$$
T x=\sum_{j=1}^{\infty}\left\langle x, b_{j}\right\rangle a_{j}, \quad x \in H .
$$

Sometimes these operators are referred to as trace class operators. It is well known that $\mathcal{L}_{1}(H)$ becomes a Banach space under the norm

$$
\|T\|_{\mathrm{Tr}}=\inf \left\{\sum_{j=1}^{\infty}\left\|a_{j}\right\|\left\|b_{j}\right\|: T x=\sum_{j=1}^{\infty}\left\langle x, b_{j}\right\rangle a_{j}\right\} .
$$


If $T \in \mathcal{L}_{1}(H)$, then for any orthonormal basis $\left\{e_{k}\right\}_{k=1}^{\infty} \subset H$ the trace of $T$, defined as

$$
\operatorname{Tr}(T)=\sum_{k=1}^{\infty}\left\langle T e_{k}, e_{k}\right\rangle
$$

is finite and the sum is independent of the choice of the orthonormal basis. If $T \geq 0$ (selfadjoint, positive semidefinite) and the sum in (2.4) converges for one particular orthonormal basis, then $T \in \mathcal{L}_{1}(H)$. We recall the following well known properties of the trace and trace norm which we frequently use, see [1, App. C], [12, Chapt. 30] and [15. Chapt. 7]. If $S \in \mathcal{B}(H)$ and $T \in \mathcal{L}_{1}(H)$, then both $T S$ and $S T$ belong to $\mathcal{L}_{1}(H)$ and

$$
\begin{aligned}
& \operatorname{Tr}(T S)=\operatorname{Tr}(S T), \\
& |\operatorname{Tr}(T S)|=|\operatorname{Tr}(S T)| \leq\|T\|_{\operatorname{Tr}}\|S\|_{\mathcal{B}(H)}, \\
& \|T S\|_{\operatorname{Tr}} \leq\|T\|_{\operatorname{Tr}}\|S\|_{\mathcal{B}(H)}, \quad\|S T\|_{\operatorname{Tr}} \leq\|T\|_{\operatorname{Tr}}\|S\|_{\mathcal{B}(H)} .
\end{aligned}
$$

Furthermore, if $T \in \mathcal{L}_{1}(H)$, then its adjoint $T^{*} \in \mathcal{L}_{1}(H)$ and

$$
\operatorname{Tr}(T)=\operatorname{Tr}\left(T^{*}\right), \quad\|T\|_{\operatorname{Tr}}=\left\|T^{*}\right\|_{\operatorname{Tr}} .
$$

If both $T, S \in \mathcal{L}_{2}(H)$, then $T S \in \mathcal{L}_{1}(H)$ and

$$
\|T S\|_{\operatorname{Tr}} \leq\|T\|_{\mathrm{HS}}\|S\|_{\mathrm{HS}} .
$$

The following theorem compares the conditions 1.8, , 1.12,, 1.14 , and 1.18 on the covariance operator $Q$. Since $\|T\|_{\mathrm{HS}}^{2}=\operatorname{Tr}\left(T^{*} T\right)=\left\|T^{*} T\right\|_{\mathrm{Tr}}$, we note that 1.8 is expressed as the trace of a symmetric, positive semidefinite operator:

$$
\left\|\Lambda^{\frac{\beta-1}{2}} Q^{\frac{1}{2}}\right\|_{\mathrm{HS}}^{2}=\operatorname{Tr}\left(\left[\Lambda^{\frac{\beta-1}{2}} Q^{\frac{1}{2}}\right]^{*} \Lambda^{\frac{\beta-1}{2}} Q^{\frac{1}{2}}\right)=\left\|\left[\Lambda^{\frac{\beta-1}{2}} Q^{\frac{1}{2}}\right]^{*} \Lambda^{\frac{\beta-1}{2}} Q^{\frac{1}{2}}\right\|_{\operatorname{Tr}},
$$

while 1.12 and 1.18 involve the trace norm of a nonsymmetric operator.

Theorem 2.1. Assume that $Q \in \mathcal{B}(H)$ is selfadjoint, positive semidefinite and that $A$ is a densely defined, unbounded, selfadjoint, positive definite, linear operator on $H$ with an orthonormal basis of eigenvectors. Then the following inequalities hold, for $s \in \mathbb{R}, \alpha>0$,

$$
\begin{aligned}
& \left\|A^{\frac{s}{2}} Q^{\frac{1}{2}}\right\|_{\mathrm{HS}}^{2} \leq\left\|A^{s} Q\right\|_{\operatorname{Tr}} \leq\left\|A^{s+\alpha} Q\right\|_{\mathcal{B}(H)}\|\| A^{-\alpha} \|_{\operatorname{Tr}}, \\
& \left\|A^{\frac{s}{2}} Q^{\frac{1}{2}}\right\|_{\mathrm{HS}}^{2} \leq\left\|A^{s+\frac{1}{2}} Q A^{-\frac{1}{2}}\right\|_{\mathrm{Tr}},
\end{aligned}
$$

provided that the respective norms are finite. Furthermore, if $A$ and $Q$ have a common basis of eigenvectors, in particular, if $Q=I$, then

$$
\left\|A^{\frac{s}{2}} Q^{\frac{1}{2}}\right\|_{\mathrm{HS}}^{2}=\left\|A^{s} Q\right\|_{\operatorname{Tr}}=\left\|A^{s+\frac{1}{2}} Q A^{-\frac{1}{2}}\right\|_{\operatorname{Tr}} .
$$

Proof. If $\left\{\left(\lambda_{k}, \phi_{k}\right)\right\}_{k=1}^{\infty}$ denotes a set of eigenpairs of $A$ with orthonormal eigenvectors, then we define

$$
A^{s} x=\sum_{k=1}^{\infty} \lambda_{k}^{s}\left\langle x, \phi_{k}\right\rangle \phi_{k} .
$$


Although $\left[A^{\frac{s}{2}} Q^{\frac{1}{2}}\right]^{*}$ is not equal to $Q^{\frac{1}{2}} A^{\frac{s}{2}}$ in general, we do have $\left[A^{\frac{s}{2}} Q^{\frac{1}{2}}\right]^{*} \phi_{k}=$ $Q^{\frac{1}{2}} A^{\frac{s}{2}} \phi_{k}$, and we compute using (2.2), 2.1), 2.4), 2.6, and 2.7),

$$
\begin{aligned}
\left\|A^{\frac{s}{2}} Q^{\frac{1}{2}}\right\|_{\mathrm{HS}}^{2} & =\left\|\left[A^{\frac{s}{2}} Q^{\frac{1}{2}}\right]^{*}\right\|_{\mathrm{HS}}^{2}=\sum_{k=1}^{\infty}\left\|\left[A^{\frac{s}{2}} Q^{\frac{1}{2}}\right]^{*} \phi_{k}\right\|^{2}=\sum_{k=1}^{\infty}\left\|Q^{\frac{1}{2}} A^{\frac{s}{2}} \phi_{k}\right\|^{2} \\
& =\sum_{k=1}^{\infty} \lambda_{k}^{s}\left\|Q^{\frac{1}{2}} \phi_{k}\right\|^{2}=\sum_{k=1}^{\infty} \lambda_{k}^{s}\left\langle Q \phi_{k}, \phi_{k}\right\rangle=\sum_{k=1}^{\infty}\left\langle Q \phi_{k}, A^{s} \phi_{k}\right\rangle \\
& =\sum_{k=1}^{\infty}\left\langle A^{s} Q \phi_{k}, \phi_{k}\right\rangle=\operatorname{Tr}\left(A^{s} Q\right) \leq\left\|A^{s} Q\right\|_{\operatorname{Tr}} \leq\left\|A^{s+\alpha} Q\right\|_{\mathcal{B}(H)}\left\|A^{-\alpha}\right\|_{\operatorname{Tr}} .
\end{aligned}
$$

This is 2.10. Similarly, 2.11) is proved by

$$
\begin{aligned}
\left\|A^{\frac{s}{2}} Q^{\frac{1}{2}}\right\|_{\mathrm{HS}}^{2} & =\sum_{k=1}^{\infty} \lambda_{k}^{s}\left\langle Q \phi_{k}, \phi_{k}\right\rangle=\sum_{k=1}^{\infty}\left\langle Q \lambda_{k}^{-\frac{1}{2}} \phi_{k}, \lambda_{k}^{s+\frac{1}{2}} \phi_{k}\right\rangle \\
& =\sum_{k=1}^{\infty}\left\langle A^{s+\frac{1}{2}} Q A^{-\frac{1}{2}} \phi_{k}, \phi_{k}\right\rangle=\operatorname{Tr}\left(A^{s+\frac{1}{2}} Q A^{-\frac{1}{2}}\right) \leq\left\|A^{s+\frac{1}{2}} Q A^{-\frac{1}{2}}\right\|_{\operatorname{Tr}} .
\end{aligned}
$$

To show 2.12 we assume that $Q$ has the same eigenvectors $\phi_{k}$ with eigenvalues $\gamma_{k}$. Then

$$
A^{s} Q x=\sum_{k=1}^{\infty} \lambda_{k}^{s} \gamma_{k}\left\langle x, \phi_{k}\right\rangle \phi_{k}
$$

and hence

$$
\left\|A^{s} Q\right\|_{\operatorname{Tr}} \leq \sum_{k=1}^{\infty} \lambda_{k}^{s} \gamma_{k}=\sum_{k=1}^{\infty}\left\|A^{\frac{s}{2}} Q^{\frac{1}{2}} \phi_{k}\right\|^{2}=\left\|A^{\frac{s}{2}} Q^{\frac{1}{2}}\right\|_{\mathrm{HS}}^{2},
$$

which shows the first equality in $(2.12)$ in view of $(2.10)$. The second equality in 2.12 can be shown in a similar fashion.

Finally, we define $C_{\mathrm{b}}^{2}(H, \mathbb{R})$ to be the set of all real-valued, twice Fréchet differentiable functions $G$, whose first and second derivatives are continuous and bounded. By the Riesz representation theorem, we may identify the first derivative $D G(x)$ at $x \in H$ with an element $G^{\prime}(x) \in H$ such that

$$
D G(x) y=\left\langle G^{\prime}(x), y\right\rangle, \quad y \in H,
$$

and the second derivative $D^{2} G(x)$ with a selfadjoint linear operator $G^{\prime \prime}(x) \in \mathcal{B}(H)$ such that

$$
D^{2} G(x)(y, z)=\left\langle G^{\prime \prime}(x) y, z\right\rangle, \quad y, z \in H .
$$

We say that $G \in C^{2}(H, \mathbb{R})$ if $G, G^{\prime}$, and $G^{\prime \prime}$ are continuous, that is, $G \in C(H, \mathbb{R})$, $G^{\prime} \in C(H, H)$, and $G^{\prime \prime} \in C(H, \mathcal{B}(H))$. Thus, we define

$$
C_{\mathrm{b}}^{2}(H):=\left\{G \in C^{2}(H, \mathbb{R}):\|G\|_{C_{\mathrm{b}}^{2}(H)}<\infty\right\},
$$

with the seminorm

$$
\|G\|_{C_{\mathrm{b}}^{2}(H)}:=\sup _{x \in H}\left\|G^{\prime}(x)\right\|_{H}+\sup _{x \in H}\left\|G^{\prime \prime}(x)\right\|_{\mathcal{B}(H)} .
$$

Note that we do not assume that the function $G$ itself is bounded. 


\section{ERror Representation}

In this section we derive a representation of the weak error in the general framework. In the following sections we use this to obtain the weak convergence order for finite element approximations of various equations.

If condition 3.5 below holds, then there is a unique weak solution of

$$
\mathrm{d} Y(t)=E(T-t) B \mathrm{~d} W(t), t \in(0, T] ; \quad Y(0)=E(T) X_{0},
$$

which is given by

$$
Y(t)=E(T) X_{0}+\int_{0}^{t} E(T-s) B \mathrm{~d} W(s), \quad t \in[0, T] .
$$

Notice that $X(T)=Y(T)$, where $X$ is given by 1.2 . Similarly, we define

$$
Y_{h}(t)=E_{h}(T) X_{h 0}+\int_{0}^{t} E_{h}(T-s) B_{h} \mathrm{~d} W(s), \quad t \in[0, T],
$$

and note that $X_{h}(T)=Y_{h}(T)$, where $X_{h}$ is given by (1.4). We also consider the auxiliary problem

$$
\mathrm{d} Z(t)=E(T-t) B \mathrm{~d} W(t), t \in(\tau, T] ; \quad Z(\tau)=\xi,
$$

where $\xi$ is an $\mathcal{F}_{\tau}$-measurable random variable. Its unique weak solution is given by

$$
Z(t, \tau, \xi)=\xi+\int_{\tau}^{t} E(T-s) B \mathrm{~d} W(s), \quad t \in[\tau, T] .
$$

For $G \in C_{\mathrm{b}}^{2}(H, \mathbb{R})$, we define a function $u: H \times[0, T] \rightarrow \mathbb{R}$ by

$$
u(x, t)=\mathbf{E}(G(Z(T, t, x))) .
$$

It follows from (3.1) that its partial derivatives are given by

$$
\begin{aligned}
u_{x}(x, t) & =\mathbf{E}\left(G^{\prime}(Z(T, t, x))\right), \\
u_{x x}(x, t) & =\mathbf{E}\left(G^{\prime \prime}(Z(T, t, x))\right) .
\end{aligned}
$$

It is known (see, for example, 2, Chapters 3 and 6]) that $u$ is a solution to Kolmogorov's equation

$$
\begin{aligned}
& u_{t}(x, t)+\frac{1}{2} \operatorname{Tr}\left(u_{x x}(x, t) E(T-t) B Q B^{*} E(T-t)^{*}\right)=0, \quad(x, t) \in H \times[0, T), \\
& u(x, T)=G(x), \quad x \in H .
\end{aligned}
$$

We are now ready to prove a representation formula for the weak error.

Theorem 3.1. If

$$
\operatorname{Tr}\left(\int_{0}^{T} E(t) B Q B^{*} E(t)^{*} \mathrm{~d} t\right)<\infty
$$


and $G \in C_{\mathrm{b}}^{2}(H, \mathbb{R})$, then the weak error $e_{h}(T)$ in 1.5 has the representation

$e_{h}(T)=\mathbf{E}\left(u\left(Y_{h}(0), 0\right)-u(Y(0), 0)\right)$

$$
\begin{aligned}
+ & \frac{1}{2} \mathbf{E} \int_{0}^{T} \operatorname{Tr}\left(u_{x x}\left(Y_{h}(t), t\right)\right. \\
& \left.\times\left[E_{h}(T-t) B_{h}+E(T-t) B\right] Q\left[E_{h}(T-t) B_{h}-E(T-t) B\right]^{*}\right) \mathrm{d} t \\
= & \mathbf{E}\left(u\left(Y_{h}(0), 0\right)-u(Y(0), 0)\right) \\
+ & \frac{1}{2} \mathbf{E} \int_{0}^{T} \operatorname{Tr}\left(u_{x x}\left(Y_{h}(t), t\right)\right. \\
& \left.\times\left[E_{h}(T-t) B_{h}-E(T-t) B\right] Q\left[E_{h}(T-t) B_{h}+E(T-t) B\right]^{*}\right) \mathrm{d} t .
\end{aligned}
$$

Proof. Condition (3.5) guarantees that the stochastic convolution in 1.2$)$ exists. Since $E_{h}(t) B_{h}$ acts in a finite-dimensional space, a condition analogous to (3.5) holds and hence (1.4) exists. As in [1, Theorem 9.8], if $\xi$ is $\mathcal{F}_{t}$-measurable, then

$$
u(\xi, t)=\mathbf{E}\left(G(Z(T, t, \xi)) \mid \mathcal{F}_{t}\right) .
$$

Therefore, by the law of double expectation,

$$
\mathbf{E}(u(\xi, t))=\mathbf{E}\left(\mathbf{E}\left(G(Z(T, t, \xi)) \mid \mathcal{F}_{t}\right)\right)=\mathbf{E}(G(Z(T, t, \xi))) .
$$

Thus, with $\xi=Y(0)=E(T) X_{0}$, and since $Y(T)=X(T)$,

$$
\mathbf{E}(u(Y(0), 0))=\mathbf{E}(G(Z(T, 0, Y(0)))=\mathbf{E}(G(Y(T)))=\mathbf{E}(G(X(T)))
$$

and, with $\xi=Y_{h}(T)$,

$$
\begin{aligned}
\mathbf{E}\left(u\left(Y_{h}(T), T\right)\right) & =\mathbf{E}\left(G\left(Z\left(T, T, Y_{h}(T)\right)\right)\right) \\
& =\mathbf{E}\left(G\left(Y_{h}(T)\right)\right)=\mathbf{E}\left(G\left(X_{h}(T)\right)\right) .
\end{aligned}
$$

Hence,

$$
\begin{aligned}
e_{h}(T) & =\mathbf{E}\left(G\left(X_{h}(T)\right)-G(X(T))\right)=\mathbf{E}\left(u\left(Y_{h}(T), T\right)-u(Y(0), 0)\right) \\
& =\mathbf{E}\left(u\left(Y_{h}(0), 0\right)-u(Y(0), 0)\right)+\mathbf{E}\left(u\left(Y_{h}(T), T\right)-u\left(Y_{h}(0), 0\right)\right) .
\end{aligned}
$$

Using Itô's formula for $u\left(Y_{h}(t), t\right)$ and Kolmogorov's equation (3.4) we get

$$
\begin{aligned}
\mathbf{E}( & \left.u\left(Y_{h}(T), T\right)-u\left(Y_{h}(0), 0\right)\right) \\
= & \mathbf{E} \int_{0}^{T}\left\{u_{t}\left(Y_{h}(t), t\right)\right. \\
& \left.+\frac{1}{2} \operatorname{Tr}\left(u_{x x}\left(Y_{h}(t), t\right)\left[E_{h}(T-t) B_{h}\right] Q\left[E_{h}(T-t) B_{h}\right]^{*}\right)\right\} \mathrm{d} t \\
= & \frac{1}{2} \mathbf{E} \int_{0}^{T} \operatorname{Tr}\left(u_{x x}\left(Y_{h}(t), t\right)\right. \\
& \left.\times\left\{\left[E_{h}(T-t) B_{h}\right] Q\left[E_{h}(T-t) B_{h}\right]^{*}-E(T-t) B Q B^{*} E(T-t)^{*}\right\}\right) \mathrm{d} t .
\end{aligned}
$$


(Note that $B_{h} \in \mathcal{B}(U, H)$ with $B_{h}: U \rightarrow V_{h}, E_{h}(s): V_{h} \rightarrow V_{h}$, and that we consider $E_{h}(s) B_{h}$ as an operator in $\mathcal{B}(U, H)$. Since $E_{h}(s)$ acts only on $V_{h}$ the corresponding adjoint $\left[E_{h}(s) B_{h}\right]^{*}$ is not equal to $B_{h}^{*} E_{h}(s)^{*}$.) Now consider the identity

$$
\begin{aligned}
& u_{x x}(\xi, r)\left\{\left[E_{h}(s) B_{h}\right] Q\left[E_{h}(s) B_{h}\right]^{*}-E(s) B Q B^{*} E(s)^{*}\right\} \\
& =u_{x x}(\xi, r)\left[E_{h}(s) B_{h}-E(s) B\right] Q\left[E_{h}(s) B_{h}\right]^{*} \\
& \quad+u_{x x}(\xi, r) E(s) B Q\left[E_{h}(s) B_{h}-E(s) B\right]^{*}=: S_{1}+S_{2} .
\end{aligned}
$$

The first term has finite trace since, for example, $E_{h}(s) B_{h}$ has finite trace and so has the second term, since $E(s) B Q B^{*} E(s)^{*}$ has finite trace for almost every $s$ by (3.5). Therefore, using (2.8), (2.5), and that $Q, u_{x x}(\xi, r)$ are selfadjoint, we get

$$
\begin{aligned}
\operatorname{Tr}\left(S_{1}+S_{2}\right) & =\operatorname{Tr}\left(S_{1}\right)+\operatorname{Tr}\left(S_{2}\right)=\operatorname{Tr}\left(S_{1}\right)+\operatorname{Tr}\left(S_{2}^{*}\right) \\
& =\operatorname{Tr}\left(S_{1}\right)+\operatorname{Tr}\left(\left[E_{h}(s) B_{h}-E(s) B\right] Q B^{*} E(s)^{*} u_{x x}(\xi, r)\right) \\
& =\operatorname{Tr}\left(S_{1}\right)+\operatorname{Tr}\left(u_{x x}(\xi, r)\left[E_{h}(s) B_{h}-E(s) B\right] Q B^{*} E(s)^{*}\right) \\
& =\operatorname{Tr}\left(u_{x x}(\xi, r)\left[E_{h}(s) B_{h}-E(s) B\right] Q\left[E_{h}(s) B_{h}+E(s) B\right]^{*}\right) \\
& =\operatorname{Tr}\left(\left[E_{h}(s) B_{h}+E(s) B\right] Q\left[E_{h}(s) B_{h}-E(s) B\right]^{*} u_{x x}(\xi, r)\right) \\
& =\operatorname{Tr}\left(u_{x x}(\xi, r)\left[E_{h}(s) B_{h}+E(s) B\right] Q\left[E_{h}(s) B_{h}-E(s) B\right]^{*}\right) .
\end{aligned}
$$

The proof is completed by inserting (3.11) or $(3.12)$ into $(3.10)$ and using $(3.9)$.

\section{Application to Parabolic Equations}

In this section we apply the error representation in Section 3 to finite element approximations of the linear stochastic heat and Cahn-Hilliard equations.

4.1. The stochastic heat equation. Let $\mathcal{D} \subset \mathbb{R}^{d}$ be a bounded domain, let $\Lambda:=-\Delta$, where $\Delta=\sum_{k=1}^{d} \partial^{2} / \partial \xi_{k}^{2}$ is the Laplace operator, and set $D(\Lambda)=$ $H^{2}(\mathcal{D}) \cap H_{0}^{1}(\mathcal{D})$. Let $U=H:=L_{2}(\mathcal{D})$ with norm $\|\cdot\|$ and inner product $\langle\cdot, \cdot\rangle$, $B:=I$ and $A:=\Lambda$. Then (1.1) takes the form of the stochastic heat equation (1.6). In order to quantify spatial regularity we introduce the following spaces and norms. Let

$$
\dot{H}^{\alpha}:=D\left(\Lambda^{\alpha / 2}\right), \quad|v|_{\alpha}:=\left\|\Lambda^{\alpha / 2} v\right\|=\left(\sum_{j=1}^{\infty} \lambda_{j}^{\alpha}\left\langle v, \phi_{j}\right\rangle^{2}\right)^{1 / 2}, \quad \alpha \in \mathbb{R},
$$

where $\left\{\left(\lambda_{j}, \phi_{j}\right)\right\}_{j=1}^{\infty}$ are the eigenpairs of $\Lambda$ with orthonormal eigenvectors. Then $\dot{H}^{\alpha} \subset \dot{H}^{\beta}$ for $\alpha \geq \beta$. It is known that $\dot{H}^{0}=L_{2}(\mathcal{D}), \dot{H}^{1}=H_{0}^{1}(\mathcal{D}), \dot{H}^{2}=$ $H^{2}(\mathcal{D}) \cap H_{0}^{1}(\mathcal{D})$ with equivalent norms and that $\dot{H}^{-\beta}$ can be identified with the dual space $\left(\dot{H}^{\beta}\right)^{*}$ for $\beta>0$, see [13, Chapt. 3].

Let $\left\{S_{h}\right\}_{h>0}$ be a family of function spaces consisting of continuous piecewise polynomials of degree $\leq r-1$ with respect to a family of triangulations of $\mathcal{D}$ and such that $S_{h} \subset H_{0}^{1}(D)$. The parameter $h$ is the maximal mesh size of the triangulation and $r$ may be referred to as the order of the finite element method. Let $P_{h}: H \rightarrow S_{h}$ denote the orthogonal projection and let $\Lambda_{h}: S_{h} \rightarrow S_{h}$ be the "discrete Laplacian" defined by

$$
\left\langle\Lambda_{h} \psi, \chi\right\rangle=\langle\nabla \psi, \nabla \chi\rangle, \quad \forall \psi, \chi \in S_{h}
$$


Our basic assumption on the finite element method is that the Ritz projection $R_{h}: \dot{H}^{1} \rightarrow S_{h}$ defined as

$$
\left\langle\nabla R_{h} v, \nabla \chi\right\rangle=\langle\nabla v, \nabla \chi\rangle, \quad \forall v \in \dot{H}^{1}, \chi \in S_{h},
$$

satisfies the error bound

$$
\left\|R_{h} v-v\right\| \leq C h^{\beta}|v|_{\beta}, \quad v \in \dot{H}^{\beta}, 1 \leq \beta \leq r .
$$

This holds, for example, with $r=2$ if $\mathcal{D}$ is a convex polygonal domain and $S_{h}$ consists of piecewise linear functions. See 13 for further details.

If we set $V_{h}:=S_{h}, B_{h}:=P_{h}, A_{h}:=\Lambda_{h}$, and $X_{h 0}:=P_{h} X_{0}$, then (1.4) takes the form of the semidiscrete finite element approximation (1.7). We have the following result for the weak error.

Theorem 4.1. Let $X$ and $X_{h}$ be the solutions of (1.6) and (1.7), respectively. Let $g \in C_{\mathrm{b}}^{2}(H, \mathbb{R})$ and assume that $\left\|A^{\frac{\beta-1}{2}} Q^{\frac{1}{2}}\right\|_{\mathrm{HS}}=\left\|\Lambda^{\frac{\beta-1}{2}} Q^{\frac{1}{2}}\right\|_{\mathrm{HS}}<\infty$ for some $\beta \in(0,1]$. Then there are $C>0, h_{0}>0$, depending on $g, X_{0}, Q, \beta$, and $T$ but not on $h$, such that for $h \leq h_{0}$,

$$
\left|\mathbf{E}\left(g\left(X_{h}(T)\right)-g(X(T))\right)\right| \leq C h^{2 \beta}|\log (h)| .
$$

If, in addition $X_{0} \in L_{1}\left(\Omega, \dot{H}^{2 \beta}\right)$, then $C$ is independent of $T$ as well.

Proof. If $\left\|A^{\frac{\beta-1}{2}} Q^{\frac{1}{2}}\right\|_{\mathrm{HS}}<\infty$ for some $\beta \in(0,1]$, then 3.5$)$ holds, see [14. This guarantees that $X(t)$ and $X_{h}(t)$ are defined. Let $F_{h}(t):=E_{h}(t) P_{h}-E(t)$ be the deterministic error operator with $h \leq h_{0}$ small enough. We recall the error estimates, see [13, Chapt. 3],

$$
\left\|F_{h}(t) v\right\| \leq C h^{s} t^{-\frac{s-\gamma}{2}}|v|_{\gamma}, \quad 0 \leq \gamma \leq s \leq r .
$$

We use Theorem 3.1 to estimate the weak error with $G:=g$. First, by the chain rule, and $Y_{h}(0)-Y(0)=E_{h}(T) P_{h} X_{0}-E(T) X_{0}=F_{h}(T) X_{0}$,

$$
\begin{aligned}
\mathbf{E} & \left(u\left(Y_{h}(0), 0\right)-u(Y(0), 0)\right) \\
& =\mathbf{E} \int_{0}^{1}\left\langle u_{x}\left(Y(0)+s\left(Y_{h}(0)-Y(0)\right), 0\right), Y_{h}(0)-Y(0)\right\rangle \mathrm{d} s \\
& =\mathbf{E} \int_{0}^{1}\left\langle u_{x}\left(E(T) X_{0}+s F_{h}(T) X_{0}, 0\right), F_{h}(T) X_{0}\right\rangle \mathrm{d} s .
\end{aligned}
$$

Thus, using 3.2 and 4.3 , we obtain

$$
\begin{aligned}
& \left|\mathbf{E}\left(u\left(Y_{h}(0), 0\right)-u(Y(0), 0)\right)\right| \leq \sup _{x \in H}\left\|u_{x}(x, 0)\right\| \mathbf{E}\left(\left\|F_{h}(T) X_{0}\right\|\right) \\
& \quad \leq C h^{2 \beta} T^{-\frac{2 \beta-\gamma}{2}} \mathbf{E}\left(\left|X_{0}\right|_{\gamma}\right) \sup _{x \in H}\left\|g^{\prime}(x)\right\|, \quad 0 \leq \gamma \leq 2 \beta .
\end{aligned}
$$

If $\gamma=2 \beta$ there is no dependence on $T$. Next, we estimate the second term 3.6 in the error representation in Theorem 3.1. Since $E(t), E_{h}(t) P_{h}$, and hence also 
$F_{h}(t)$, are selfadjoint, we obtain by means of 2.6, 2.7), 2.8), and 2.9,

$$
\begin{aligned}
\mid \mathbf{E} \int_{0}^{T} & \operatorname{Tr}\left(u_{x x}\left(Y_{h}(t), t\right)\right. \\
& \left.\times\left[E_{h}(T-t) B_{h}+E(T-t) B\right] Q\left[E_{h}(T-t) B_{h}-E(T-t) B\right]^{*}\right) \mathrm{d} t \mid \\
= & \mid \mathbf{E} \int_{0}^{T} \operatorname{Tr}\left(u_{x x}\left(Y_{h}(t), t\right)\left[E_{h}(T-t) P_{h}+E(T-t)\right]^{*}\right. \\
& \left.\times A^{\frac{1-\beta}{2}} A^{\frac{\beta-1}{2}} Q^{\frac{1}{2}} Q^{\frac{1}{2}} A^{\frac{\beta-1}{2}} A^{\frac{1-\beta}{2}} F_{h}(T-t)\right) \mathrm{d} t \mid \\
= & \mid \mathbf{E} \int_{0}^{T} \operatorname{Tr}\left(u_{x x}\left(Y_{h}(t), t\right)\left(A^{\frac{1-\beta}{2}}\left[E_{h}(T-t) P_{h}+E(T-t)\right]\right)^{*}\right. \\
& \left.\times A^{\frac{\beta-1}{2}} Q^{\frac{1}{2}} Q^{\frac{1}{2}} A^{\frac{\beta-1}{2}} A^{\frac{1-\beta}{2}} F_{h}(T-t)\right) \mathrm{d} t \mid \\
\leq & \mathbf{E} \int_{0}^{T}\left\|u_{x x}\left(Y_{h}(t), t\right)\left(A^{\frac{1-\beta}{2}}\left[E_{h}(T-t) P_{h}+E(T-t)\right]\right)^{*} A^{\frac{\beta-1}{2}} Q^{\frac{1}{2}}\right\|_{\mathrm{HS}} \\
& \times\left\|Q^{\frac{1}{2}} A^{\frac{\beta-1}{2}} A^{\frac{1-\beta}{2}} F_{h}(T-t)\right\|_{\mathrm{HS}} \mathrm{d} t \\
\leq & \sup _{(x, t) \in H \times[0, T]}\left\|u_{x x}(x, t)\right\|_{\mathcal{B}(H)}\left\|A^{\frac{\beta-1}{2}} Q^{\frac{1}{2}}\right\|_{\mathrm{HS}}^{2} \\
& \times \int_{0}^{T}\left\|A^{\frac{1-\beta}{2}}\left(E_{h}(t) P_{h}+E(t)\right)\right\|_{\mathcal{B}(H)}\left\|A^{\frac{1-\beta}{2}} F_{h}(t)\right\|_{\mathcal{B}(H)} \mathrm{d} t .
\end{aligned}
$$

Since $\left\|A^{\frac{1}{2}} v_{h}\right\|=\left\|\nabla v_{h}\right\|=\left\|A_{h}^{\frac{1}{2}} v_{h}\right\|$ for $v_{h} \in S_{h}$, we conclude $\left\|A^{\delta} v_{h}\right\| \leq\left\|A_{h}^{\delta} v_{h}\right\|$ for $v_{h} \in S_{h}, \delta \in\left[0, \frac{1}{2}\right]$, and using also the analyticity of the semigroups we have

$$
\left\|A^{\delta}\left(E_{h}(t) P_{h}+E(t)\right)\right\|_{\mathcal{B}(H)} \leq C e^{-\omega t} t^{-\delta}, \quad \delta \in\left[0, \frac{1}{2}\right] .
$$

To estimate $\left\|A^{\frac{1-\beta}{2}} F_{h}(t)\right\|_{\mathcal{B}(H)}$ we use interpolation. By analyticity, as above,

$$
\left\|A^{\delta} F_{h}(t)\right\|_{\mathcal{B}(H)} \leq C t^{-\delta}, \quad \delta \in\left[0, \frac{1}{2}\right] .
$$

Interpolation between 4.7) with $\delta=\frac{1}{2}$ and 4.3 with $s=2$ and $\gamma=0$ yields

$$
\left\|A^{\frac{1-\beta}{2}} F_{h}(t)\right\|_{\mathcal{B}(H)} \leq\left\|F_{h}(t)\right\|_{\mathcal{B}(H)}^{\beta}\left\|A^{\frac{1}{2}} F_{h}(t)\right\|_{\mathcal{B}(H)}^{1-\beta} \leq C h^{2 \beta} t^{-\frac{1+\beta}{2}}, \quad \beta \in[0,1] .
$$

Therefore, for $\beta \in(0,1]$ one may estimate the integral in 4.5 as follows

$$
\begin{aligned}
& \int_{0}^{T}\left\|A^{\frac{1-\beta}{2}}\left(E_{h}(t) P_{h}+E(t)\right)\right\|_{\mathcal{B}(H)}\left\|A^{\frac{1-\beta}{2}} F_{h}(t)\right\|_{\mathcal{B}(H)} \mathrm{d} t \\
& =\left(\int_{0}^{h^{2}}+\int_{h^{2}}^{T}\right)\left\|A^{\frac{1-\beta}{2}}\left(E_{h}(t) P_{h}+E(t)\right)\right\|_{\mathcal{B}(H)}\left\|A^{\frac{1-\beta}{2}} F_{h}(t)\right\|_{\mathcal{B}(H)} \mathrm{d} t \\
& \leq C \int_{0}^{h^{2}} t^{-\frac{1-\beta}{2}} t^{-\frac{1-\beta}{2}} \mathrm{~d} t+C \int_{h^{2}}^{T} e^{-\omega t} t^{-\frac{1-\beta}{2}} h^{2 \beta} t^{-\frac{1+\beta}{2}} \mathrm{~d} t \leq C h^{2 \beta}|\log (h)| .
\end{aligned}
$$

Finally, using (3.3), we obtain

$$
\sup _{(x, t) \in H \times[0, T]}\left\|u_{x x}(x, t)\right\|_{\mathcal{B}(H)} \leq \sup _{x \in H}\left\|g^{\prime \prime}(x)\right\|_{\mathcal{B}(H)},
$$

and the proof is complete in view of 4.4 . 
By inspection of the above proof we see that the error estimate is

$$
\begin{aligned}
\left|\mathbf{E}\left(g\left(X_{h}(T)\right)-g(X(T))\right)\right| \leq & C h^{2 \beta} T^{-\frac{2 \beta-\gamma}{2}} \mathbf{E}\left(\left|X_{0}\right|_{\gamma}\right) \sup _{x \in H}\left\|g^{\prime}(x)\right\| \\
& +C h^{2 \beta}|\log (h)| \beta^{-1} \sup _{x \in H}\left\|g^{\prime \prime}(x)\right\|\left\|A^{\frac{\beta-1}{2}} Q^{\frac{1}{2}}\right\|_{\mathrm{HS}}^{2} .
\end{aligned}
$$

Similar remarks can be made about the theorems to follow.

Theorem 4.1 does not allow $\beta>1$. This is satisfactory if $r=2$, but for higher order elements, that is, $r>2$, it is insufficient. Under a slightly stronger condition on $A$ and $Q$ we now extend the result to the case $\beta>1$.

Theorem 4.2. Let $X$ and $X_{h}$ be the solutions of $(1.6)$ and (1.7), respectively. Let $g \in C_{\mathrm{b}}^{2}(H, \mathbb{R})$ and assume that $\left\|A^{\beta-1} Q\right\|_{\mathrm{Tr}}=\left\|\Lambda^{\beta-1} Q\right\|_{\mathrm{Tr}}<\infty$ for some $\beta \in\left[1, \frac{r}{2}\right]$. Then there are $C>0, h_{0}>0$, depending on $g, X_{0}, Q, \beta$, and $T$ but not on $h$, such that for $h \leq h_{0}$,

$$
\left|\mathbf{E}\left(g\left(X_{h}(T)\right)-g(X(T))\right)\right| \leq C h^{2 \beta}|\log (h)| .
$$

If, in addition $X_{0} \in L_{1}\left(\Omega, \dot{H}^{2 \beta}\right)$, then $C$ is independent of $T$ as well.

Proof. If $\left\|A^{\beta-1} Q\right\|_{\operatorname{Tr}}<\infty$ for some $\beta \in\left[1, \frac{r}{2}\right]$, then (3.5) holds by Theorem 2.1 and [14, so that $X(t)$ and $X_{h}(t)$ are defined. From (4.3) with $\gamma=2 \beta-2 \leq s=2 \beta$ it follows that

$$
\left\|F_{h}(t) A^{1-\beta}\right\|_{\mathcal{B}(H)} \leq C h^{2 \beta} t^{-1}, \quad 1 \leq \beta \leq \frac{r}{2}
$$

and, by 4.7 and since $\beta \geq 1$,

$$
\left\|F_{h}(t) A^{1-\beta}\right\|_{\mathcal{B}(H)} \leq\left\|F_{h}(t)\right\|_{\mathcal{B}(H)}\left\|A^{1-\beta}\right\|_{\mathcal{B}(H)} \leq C
$$

The first term in the error representation in Theorem 3.1 can be estimated the same way as in Theorem 4.1. To bound the second term (3.7) we use (4.6) with $\delta=0$ and 2.5 to obtain

$$
\begin{aligned}
\mid \mathbf{E} \int_{0}^{T} & \operatorname{Tr}\left(u_{x x}\left(Y_{h}(t), t\right)\right. \\
& \left.\times\left[E_{h}(T-t) B_{h}-E(T-t) B\right] Q\left[E_{h}(T-t) B_{h}+E(T-t) B\right]^{*}\right) \mathrm{d} t \mid \\
= & \mid \mathbf{E} \int_{0}^{T} \operatorname{Tr}\left(u_{x x}\left(Y_{h}(t), t\right)\right. \\
& \left.\times F_{h}(t) A^{1-\beta} A^{\beta-1} Q\left[E_{h}(T-t) P_{h}+E(T-t)\right]^{*}\right) \mathrm{d} t \mid \\
\leq & C \sup _{(x, t) \in H \times[0, T]}\left\|u_{x x}(x, t)\right\|_{\mathcal{B}(H)}\left\|A^{\beta-1} Q\right\|_{\operatorname{Tr}} \int_{0}^{T}\left\|F_{h}(t) A^{\beta-1}\right\|_{\mathcal{B}(H)} e^{-\omega t} \mathrm{~d} t
\end{aligned}
$$

Using 4.9 and 4.10 we now have

$$
\begin{aligned}
& \int_{0}^{T}\left\|F_{h}(t) A^{1-\beta}\right\|_{\mathcal{B}(H)} e^{-\omega t} \mathrm{~d} t=\left(\int_{0}^{h^{2 \beta}}+\int_{h^{2 \beta}}^{T}\right)\left\|F_{h}(t) A^{1-\beta}\right\|_{\mathcal{B}(H)} e^{-\omega t} \mathrm{~d} t \\
& \leq C \int_{0}^{h^{2 \beta}} \mathrm{d} t+C h^{2 \beta} \int_{h^{2 \beta}}^{T} t^{-1} e^{-\omega t} \mathrm{~d} t \leq C h^{2 \beta}|\log (h)|,
\end{aligned}
$$

and the proof is complete in view of 4.8 . 
In [14] the strong rate of convergence is found to be $O\left(h^{\beta}\right)$ under the condition $\left\|\Lambda^{\frac{\beta-1}{2}} Q^{\frac{1}{2}}\right\|_{\mathrm{HS}}<\infty$. Theorem 2.1 shows that $\left\|\Lambda^{\beta-1} Q\right\|_{\operatorname{Tr}}<\infty$ provides a sufficient condition for this and the conditions coincide if $\beta=1$ or if $\Lambda$ and $Q$ commute.

In the special case $Q=I$ a simple calculation using the asymptotics $\lambda_{j} \sim j^{2 / d}$, $j \rightarrow \infty$, of the eigenvalues of $\Lambda$ shows that the spatial dimension $d$ has to be 1 and $\beta<\frac{1}{2}$, which gives a weak order of almost $h$. If $\operatorname{Tr}(Q)<\infty$, then we may take $\beta=1$ and hence the rate of weak convergence is at least $O\left(h^{2}|\log (h)|\right)$.

4.2. The linear Cahn-Hilliard-Cook equation. Let $\mathcal{D}$ be a bounded domain in $\mathbb{R}^{d}$ for $d \leq 3$. Let $H=U$ be the subspace of $L_{2}(\mathcal{D})$, which is orthogonal to constants with norm $\|\cdot\|$ and inner product $\langle\cdot, \cdot\rangle$, i.e., $H=U=\left\{v \in L_{2}:\langle v, 1\rangle=0\right\}$, and let $B=I$. Let $H^{s}=H^{s}(\mathcal{D})$ be the usual Sobolev space. We define the linear operator $\Lambda:=-\Delta$ with domain of definition

$$
D(\Lambda)=\left\{v \in H^{2} \cap H: \frac{\partial v}{\partial n}=0 \text { on } \partial \mathcal{D}\right\} .
$$

Then $\Lambda$ is a selfadjoint, positive definite, densely defined operator on $H$. If we set $A:=\Lambda^{2}$, then $-A$ generates an analytic semigroup on $H$. We also define $\dot{H}^{s}=\mathcal{D}\left(\Lambda^{\frac{s}{2}}\right)$ with norms $|v|_{s}=\left\|\Lambda^{\frac{s}{2}} v\right\|$ for real $s$. It is well known that, for integer $s \geq 0, \dot{H}^{s}$ is a subspace of $H^{s} \cap H$ characterized by certain boundary conditions and that the norm $|\cdot|_{s}$ is equivalent to the standard norm $\|\cdot\|_{H^{s}}$ on $\dot{H}^{s}$. In particular, we have $\dot{H}^{1}=H^{1} \cap H$ and the norm $|v|_{1}=\left\|\Lambda^{\frac{1}{2}} v\right\|=\|\nabla v\|$ is equivalent to $\|v\|_{H^{1}}$ on $\dot{H}^{1}$. With these definitions (1.1) takes the form of the linear Cahn-Hilliard-Cook equation 1.15.

With $S_{h} \subset H^{1}$ being a family of finite dimensional subspaces we set $V_{h}:=\{\chi \in$ $\left.S_{h}:\langle\chi, 1\rangle=0\right\}$ and define $\Lambda_{h}: V_{h} \rightarrow V_{h}$ by

$$
\left\langle\Lambda_{h} \chi, \eta\right\rangle=\langle\nabla \chi, \nabla \eta\rangle, \quad \chi, \eta \in V_{h} .
$$

Finally, we set $A_{h}:=\Lambda_{h}^{2}, B_{h}:=P_{h}: H \rightarrow V_{h}$ the orthogonal projection, and set $X_{h 0}:=P_{h} X_{0}$. Then 1.3 takes the form 1.16 .

As for the heat equation, we assume that the Ritz projection $R_{h}: \dot{H}^{1} \rightarrow V_{h}$ defined as in 4.1 satisfies an error estimate of the form 4.2. This holds, for example, with $r=2$ if $\mathcal{D}$ is a convex polygonal domain and with $S_{h}$ being the standard family of finite element spaces consisting of continuous piecewise linear functions on a regular family of triangulations of $\mathcal{D}$ with maximum mesh size $h$.

Theorem 4.3. Let $X$ and $X_{h}$ be the solutions of 1.15 and 1.16 , respectively. Let $g \in C_{\mathrm{b}}^{2}(H, \mathbb{R})$, assume that $0<\beta \leq \min \left(2, \frac{r}{2}\right)$, and, for some $K$,

$$
\begin{aligned}
& \left\|A^{\frac{\beta-2}{2}} Q\right\|_{\operatorname{Tr}}=\left\|\Lambda^{\beta-2} Q\right\|_{\operatorname{Tr}} \leq K, \\
& \left\|A_{h}^{\frac{\beta-2}{2}} P_{h} Q\right\|_{\operatorname{Tr}}=\left\|\Lambda_{h}^{\beta-2} P_{h} Q\right\|_{\operatorname{Tr}} \leq K, \quad 0<h \leq 1 .
\end{aligned}
$$

Then there are $C>0, h_{0}>0$, depending on $g, X_{0}, K, \beta$, and $T$ but not on $h$, such that for $h \leq h_{0}$,

$$
\left|\mathbf{E}\left(g\left(X_{h}(T)\right)-g(X(T))\right)\right| \leq C h^{2 \beta}|\log (h)| .
$$

If, in addition $X_{0} \in L_{1}\left(\Omega, \dot{H}^{2 \beta}\right)$, then $C$ is independent of $T$ as well. 
M. KOVÁCS, S. LARSSON, AND F. LINDGREN

Proof. If $\left\|A^{\frac{\beta-2}{2}} Q\right\|_{\operatorname{Tr}}<\infty$ for some $\beta \geq 0$, then (3.5) holds, see [11, and $X$ and $X_{h}$ exist. Let $F_{h}(t):=E_{h}(t) P_{h}-E(t)$ be the deterministic error operator with $h \leq h_{0}$ small enough. We recall from [6] the error estimate

$$
\left\|F_{h}(t) v\right\| \leq C h^{s} t^{-\frac{s-\gamma}{4}}|v|_{\gamma}, \quad 0 \leq \gamma \leq s \leq r .
$$

We use Theorem 3.1 to estimate the weak error. As in the proof of Theorem 4.1 we get, for $0 \leq \gamma \leq 2 \beta \leq r$,

$$
\left|\mathbf{E}\left(u\left(Y_{h}(0), 0\right)-u(Y(0), 0)\right)\right| \leq C h^{2 \beta} T^{-\frac{2 \beta-\gamma}{4}} \mathbf{E}\left(\left|X_{0}\right|_{\gamma}\right) \sup _{x \in H}\left\|g^{\prime}(x)\right\| .
$$

To estimate the second term (3.6) in the error representation we proceed as in (4.4), 4.5. By inserting both $A^{ \pm \frac{\beta-2}{2}}=\Lambda^{ \pm(\beta-2)}$ and $A_{h}^{ \pm \frac{\beta-2}{2}}=\Lambda_{h}^{ \pm(\beta-2)}$, we obtain this time

$$
\begin{aligned}
\mid \mathbf{E} \int_{0}^{T} & \operatorname{Tr}\left(u_{x x}\left(Y_{h}(t), t\right)\right. \\
& \left.\times\left[E_{h}(T-t) B_{h}+E(T-t) B\right] Q\left[E_{h}(T-t) B_{h}-E(T-t) B\right]^{*}\right) \mathrm{d} t \mid \\
= & \mid \mathbf{E} \int_{0}^{T} \operatorname{Tr}\left(u _ { x x } ( Y _ { h } ( t ) , t ) \left[A_{h}^{\frac{2-\beta}{2}} E_{h}(T-t) A_{h}^{\frac{\beta-2}{2}} P_{h}\right.\right. \\
& \left.\left.+A^{\frac{2-\beta}{2}} E(T-t) A^{\frac{\beta-2}{2}}\right] Q F_{h}(T-t)^{*}\right) \mathrm{d} t \mid \\
\leq & \sup _{(x, t) \in H \times[0, T]}\left\|u_{x x}(x, t)\right\|_{\mathcal{B}(H)}\left(\left\|A_{h}^{\frac{\beta-2}{2}} P_{h} Q\right\|_{\operatorname{Tr}}+\left\|A^{\frac{\beta-2}{2}} Q\right\|_{\operatorname{Tr}}\right) \\
& \times \int_{0}^{T}\left(\left\|A_{h}^{\frac{2-\beta}{2}} E_{h}(t) P_{h}\right\|_{\mathcal{B}(H)}+\left\|A^{\frac{2-\beta}{2}} E(t)\right\|_{\mathcal{B}(H)}\right)\left\|F_{h}(t)\right\|_{\mathcal{B}(H)} \mathrm{d} t .
\end{aligned}
$$

In view of 4.8 and 4.11 , 4.12 it remains to bound the integral. By analyticity of the semigroups we have

$$
\left\|A_{h}^{\delta}\left(E_{h}(t) P_{h}\left\|_{\mathcal{B}(H)}+\right\| A^{\delta} E(t)\right)\right\|_{\mathcal{B}(H)} \leq C e^{-\omega t} t^{-\delta}, \quad \delta \geq 0 .
$$

Therefore, by using 4.14 with $\delta=\frac{2-\beta}{2} \in[0,1)$, that is, $\beta \in(0,2]$, and 4.13 with $s=0$ and $s=2 \beta \leq r, \gamma=0$,

$$
\begin{aligned}
\int_{0}^{T} & \left(\left\|A_{h}^{\frac{2-\beta}{2}} E_{h}(t) P_{h}\right\|_{\mathcal{B}(H)}+\left\|A^{\frac{2-\beta}{2}} E(t)\right\|_{\mathcal{B}(H)}\right)\left\|F_{h}(t)\right\|_{\mathcal{B}(H)} \mathrm{d} t \\
= & \left(\int_{0}^{h^{4}}+\int_{h^{4}}^{T}\right)\left\|A^{\frac{2-\beta}{2}}\left[E_{h}(t) P_{h}+E(t)\right]\right\|_{\mathcal{B}(H)}\left\|F_{h}(t)\right\|_{\mathcal{B}(H)} \mathrm{d} t \\
& \leq C \int_{0}^{h^{4}} t^{-\frac{2-\beta}{2}} \mathrm{~d} t+C \int_{h^{4}}^{T} e^{-\omega t} t^{-\frac{2-\beta}{2}} h^{2 \beta} t^{-\frac{2 \beta}{4}} \mathrm{~d} t \leq C h^{2 \beta}|\log (h)| .
\end{aligned}
$$

This completes the proof.

In [11] the strong rate of convergence is found to be $O\left(h^{\beta}|\log (h)|\right)$ under the condition $\left\|A^{\frac{\beta-2}{4}} Q^{\frac{1}{2}}\right\|_{\text {HS }}<\infty$. Theorem 2.1 shows that 4.11) provides a sufficient condition for this and that the conditions coincide if $A$ and $Q$ commute or if $\beta=2$, that is, $\operatorname{Tr}(Q)<\infty$.

It remains to identify conditions under which we have 4.12 together with (4.11). This is addressed in the next theorem. 
Theorem 4.4. Let $X$ and $X_{h}$ be the solutions of (1.15) and (1.16), respectively. Let $g \in C_{\mathrm{b}}^{2}(H, \mathbb{R})$ and make one of the following assumptions.

(i) Assume that $Q=I, 0<\beta \leq \min \left(2, \frac{r}{2}\right)$, and $\left\|\Lambda^{\beta-2}\right\|_{\operatorname{Tr}}<\infty$.

(ii) Assume that $\operatorname{Tr}(Q)<\infty, r=4$, and $\beta=2$.

(iii) Assume that $r \geq 3, \frac{3}{2} \leq \beta \leq \min \left(2, \frac{r}{2}\right)$, and $\left\|\Lambda^{\beta-2} Q\right\|_{\operatorname{Tr}}<\infty$.

(iv) Assume that $S_{h}$ is based on a quasi-uniform mesh family and that, for some $\alpha>0$, we have $0<\beta \leq \min \left(2, \frac{r}{2}\right), 0 \leq \beta-2+\alpha \leq 1$ and

$$
\left\|\Lambda^{\beta-2+\alpha} Q\right\|_{\mathcal{B}(H)}<\infty, \quad\left\|\Lambda^{-\alpha}\right\|_{\operatorname{Tr}}<\infty .
$$

Then there are $C>0, h_{0}>0$, depending on $g, X_{0}, Q, \beta$, and $T$ but not on $h$, such that for $h \leq h_{0}$,

$$
\left|\mathbf{E}\left(g\left(X_{h}(T)\right)-g(X(T))\right)\right| \leq C h^{2 \beta}|\log (h)| .
$$

If, in addition $X_{0} \in L_{1}\left(\Omega, \dot{H}^{2 \beta}\right)$, then $C$ is independent of $T$ as well.

Proof. We must show that 4.11) and 4.12 hold in each of the four cases.

(i) The eigenvalues of $\Lambda_{h}$ and $\Lambda$ satisfy $\lambda_{h, j} \geq \lambda_{j}$ and $\left\|P_{h}\right\|_{\mathcal{B}(H)} \leq 1$, so that

$$
\left\|\Lambda_{h}^{-\alpha} P_{h}\right\|_{\operatorname{Tr}} \leq\left\|\Lambda_{h}^{-\alpha}\right\|_{\operatorname{Tr}}=\sum_{j=1}^{N_{h}} \lambda_{h, j}^{-\alpha} \leq \sum_{j=1}^{\infty} \lambda_{j}^{-\alpha}=\left\|\Lambda^{-\alpha}\right\|_{\operatorname{Tr}}, \quad \alpha \geq 0 .
$$

With $\alpha=2-\beta \geq 0$ and $Q=I$ we obtain

$$
\left\|\Lambda_{h}^{\beta-2} P_{h} Q\right\|_{\operatorname{Tr}} \leq\left\|\Lambda_{h}^{\beta-2}\right\|_{\operatorname{Tr}} \leq\left\|\Lambda^{\beta-2}\right\|_{\operatorname{Tr}}=\left\|\Lambda^{\beta-2} Q\right\|_{\operatorname{Tr}}, \quad 0<h \leq 1 .
$$

In case (ii) we have

$$
\left\|\Lambda_{h}^{\beta-2} P_{h} Q\right\|_{\operatorname{Tr}}=\left\|P_{h} Q\right\|_{\operatorname{Tr}} \leq\|Q\|_{\operatorname{Tr}}=\left\|\Lambda^{\beta-2} Q\right\|_{\operatorname{Tr}}, \quad 0<h \leq 1 .
$$

For case (iii) we use the fact that

$$
\left\|\Lambda_{h}^{-\delta} P_{h} \Lambda^{\delta}\right\| \leq C, \quad 0 \leq \delta \leq \frac{1}{2} .
$$

For $\delta=\frac{1}{2}$ this follows by using $\left\|\Lambda_{h}^{\frac{1}{2}} w_{h}\right\|=\left\|\Lambda^{\frac{1}{2}} w_{h}\right\|$ for $w_{h} \in S_{h}$ in the calculation

$$
\begin{aligned}
\left\|\Lambda_{h}^{-\frac{1}{2}} P_{h} f\right\| & =\sup _{v_{h} \in S_{h}} \frac{\left|\left\langle\Lambda_{h}^{-\frac{1}{2}} P_{h} f, v_{h}\right\rangle\right|}{\left\|v_{h}\right\|}=\sup _{v_{h} \in S_{h}} \frac{\left|\left\langle f, \Lambda_{h}^{-\frac{1}{2}} v_{h}\right\rangle\right|}{\left\|v_{h}\right\|}=\sup _{w_{h} \in S_{h}} \frac{\left|\left\langle f, w_{h}\right\rangle\right|}{\left\|\Lambda_{h}^{\frac{1}{2}} w_{h}\right\|} \\
& =\sup _{w_{h} \in S_{h}} \frac{\left|\left\langle f, w_{h}\right\rangle\right|}{\left\|\Lambda^{\frac{1}{2}} w_{h}\right\|} \leq \sup _{v \in \dot{H}^{0}} \frac{\left|\left\langle f, \Lambda^{-\frac{1}{2}} v\right\rangle\right|}{\|v\|}=\left\|\Lambda^{-\frac{1}{2}} f\right\| .
\end{aligned}
$$

The case $\delta=0$ is obvious and the general case follows by interpolation. Hence, with $\delta=2-\beta \in\left[0, \frac{1}{2}\right]$, that is, $\frac{3}{2} \leq \beta \leq 2$, we have

$$
\begin{aligned}
\left\|\Lambda_{h}^{\beta-2} P_{h} Q\right\|_{\operatorname{Tr}} & =\left\|\Lambda_{h}^{\beta-2} P_{h} \Lambda^{2-\beta} \Lambda^{\beta-2} Q\right\|_{\operatorname{Tr}} \\
& \leq\left\|\Lambda_{h}^{-(2-\beta)} P_{h} \Lambda^{2-\beta}\right\|_{\mathcal{B}(H)}\left\|\Lambda^{\beta-2} Q\right\|_{\operatorname{Tr}} \leq C\left\|\Lambda^{\beta-2} Q\right\|_{\operatorname{Tr}} .
\end{aligned}
$$

Finally, for case (iv) we first note that Theorem 2.1 shows that 4.15 implies 4.11). For quasi-uniform mesh families we have the inverse inequality $\left\|\nabla v_{h}\right\| \leq$ $C h^{-1}\left\|v_{h}\right\|, v_{h} \in S_{h}$, so that

$$
\left\|\Lambda_{h}\right\|_{\mathcal{B}(H)}=\max _{1 \leq j \leq N_{h}} \lambda_{h, j}=\max _{v_{h} \in S_{h}} \frac{\left\|\nabla v_{h}\right\|^{2}}{\left\|v_{h}\right\|^{2}} \leq C h^{-2} .
$$


Hence, using also $R_{h}=\Lambda_{h}^{-1} P_{h} \Lambda$ and 4.2 , we get

$$
\begin{aligned}
\left\|\Lambda_{h} P_{h} \Lambda^{-1} f\right\| & =\left\|\Lambda_{h} P_{h} \Lambda^{-1} f-\Lambda_{h} \Lambda_{h}^{-1} P_{h} \Lambda \Lambda^{-1} f+P_{h} f\right\| \\
& \leq\left\|\Lambda_{h} P_{h}\left(I-\Lambda_{h}^{-1} P_{h} \Lambda\right) \Lambda^{-1} f\right\|+\left\|P_{h} f\right\| \\
& \leq C h^{-2}\left\|\left(I-R_{h}\right) \Lambda^{-1} f\right\|+\|f\| \\
& \leq C h^{-2} C h^{2}\|f\|+\|f\| \leq C\|f\| .
\end{aligned}
$$

We conclude

$$
\left\|\Lambda_{h}^{\delta} P_{h} \Lambda^{-\delta}\right\|_{\mathcal{B}(H)} \leq C, \quad 0 \leq \delta \leq 1 .
$$

With $\delta=\beta-2+\alpha \in[0,1]$ and 4.16 we obtain

$$
\begin{aligned}
\left\|\Lambda_{h}^{\beta-2} P_{h} Q\right\|_{\operatorname{Tr}} & \leq\left\|\Lambda_{h}^{-\alpha}\right\|_{\operatorname{Tr}}\left\|\Lambda_{h}^{\beta-2+\alpha} P_{h} \Lambda^{-(\beta-2+\alpha)}\right\|_{\mathcal{B}(H)}\left\|\Lambda^{\beta-2+\alpha} Q\right\|_{\mathcal{B}(H)} \\
& \leq C\left\|\Lambda^{-\alpha}\right\|_{\operatorname{Tr}}\left\|\Lambda^{\beta-2+\alpha} Q\right\|_{\mathcal{B}(H)} .
\end{aligned}
$$

Finally, we comment on two of the cases of the previous theorem.

(i) A simple calculation using the asymptotics $\lambda_{j} \sim j^{2 / d}, j \rightarrow \infty$, shows that $\left\|\Lambda^{\beta-2}\right\|_{\operatorname{Tr}}<\infty$ if $\beta<2-\frac{d}{2}$.

(iv) As mentioned in (i) above, we have $\left\|\Lambda^{-\alpha}\right\|_{\operatorname{Tr}}\left\langle\infty\right.$ if $\alpha>\frac{d}{2}$ and hence it is possible to choose $\beta \in\left(0,3-\frac{d}{2}\right)$. In particular, we may have $\beta=1$ for $d=1,2,3$ and thus for $r=2$ the (almost) optimal order can be achieved in this case.

\section{Application to a hyperbolic equation}

In this section we apply the general theory to the stochastic wave equation. As for the heat equation in Subsection 4.1 we use the space $L_{2}(\mathcal{D})$ with norm $\|\cdot\|$ and inner product $\langle\cdot, \cdot\rangle$ and the Laplace operator $\Lambda=-\Delta$ with $D(\Lambda)=H^{2}(\mathcal{D}) \cap H_{0}^{1}(\mathcal{D})$. We introduce, using the notation from Subsection 4.1 .

$$
H^{\alpha}:=\dot{H}^{\alpha} \times \dot{H}^{\alpha-1}, \quad\||v|\|_{\alpha}^{2}:=\left|v_{1}\right|_{\alpha}^{2}+\left|v_{2}\right|_{\alpha-1}^{2}, \quad \alpha \in \mathbb{R},
$$

and set $H:=H^{0}=\dot{H}^{0} \times \dot{H}^{-1}$ with corresponding norm $\|\cdot \cdot\|\left|=\|\cdot \mid\|_{0}\right.$ and inner product $(\cdot, \cdot)$. We define $U:=\dot{H}^{0}=L_{2}(\mathcal{D})$ and

$$
A:=\left[\begin{array}{cc}
0 & -I \\
\Lambda & 0
\end{array}\right], \quad B:=\left[\begin{array}{l}
0 \\
I
\end{array}\right], \quad X:=\left[\begin{array}{l}
X_{1} \\
X_{2}
\end{array}\right], \quad X_{0}:=\left[\begin{array}{c}
X_{0,1} \\
X_{0,2}
\end{array}\right],
$$

with

$$
D(A)=\left\{x \in H: A x=\left[\begin{array}{c}
x_{2} \\
-\Lambda x_{1}
\end{array}\right] \in H=\dot{H}^{0} \times \dot{H}^{-1}\right\}=H^{1}=\dot{H}^{1} \times \dot{H}^{0} .
$$

Here $\Lambda$ is regarded as an operator $\dot{H}^{1} \rightarrow \dot{H}^{-1}$. The operator $-A$ is the generator of a strongly continuous semigroup $E(t)=\mathrm{e}^{-t A}$ on $H$ and

$$
E(t)=\mathrm{e}^{-t A}=\left[\begin{array}{cc}
C(t) & \Lambda^{-1 / 2} S(t) \\
-\Lambda^{1 / 2} S(t) & C(t)
\end{array}\right]
$$


where $C(t)=\cos \left(t \Lambda^{1 / 2}\right)$ and $S(t)=\sin \left(t \Lambda^{1 / 2}\right)$ are the so-called cosine and sine operators. For example, using $\left\{\left(\lambda_{j}, \phi_{j}\right)\right\}_{j=1}^{\infty}$, orthonormal eigenpairs of $\Lambda$, we have

$$
\Lambda^{-1 / 2} S(t) v=\Lambda^{-1 / 2} \sin \left(t \Lambda^{1 / 2}\right) v=\sum_{j=1}^{\infty} \lambda_{j}^{-1 / 2} \sin \left(t \lambda_{j}^{1 / 2}\right)\left\langle v, \phi_{j}\right\rangle \phi_{j} .
$$

With the above definition, the stochastic wave equation (1.17) can be written in the form of (1.1).

Let $S_{h} \subset H^{1}, \Lambda_{h}, P_{h}$ be as in Subsection 4.1 with the error estimate 4.2). The semidiscrete approximation of (1.17) is

$$
\begin{array}{ll}
\mathrm{d} X_{h, 1}-X_{h, 2} \mathrm{~d} t=0, t>0 ; & X_{h, 1}(0)=P_{h} X_{0,1}, \\
\mathrm{~d} X_{h, 2}+\Lambda_{h} X_{h, 1} \mathrm{~d} t=P_{h} \mathrm{~d} W, t>0 ; & X_{h, 2}(0)=P_{h} X_{0,2},
\end{array}
$$

We put this is the form 1.3 by defining $V_{h}:=S_{h} \times S_{h}$ and

$$
A_{h}:=\left[\begin{array}{cc}
0 & -I \\
\Lambda_{h} & 0
\end{array}\right], \quad B_{h}:=\left[\begin{array}{c}
0 \\
P_{h}
\end{array}\right], \quad X_{h 0}=P_{h} X_{0} .
$$

It can be shown that $-A_{h}$ generates a $C_{0}$-semigroup $E_{h}(t)$ given by

$$
E_{h}(t)=\mathrm{e}^{-t A_{h}}=\left[\begin{array}{cc}
C_{h}(t) & \Lambda_{h}^{-1 / 2} S_{h}(t) \\
-\Lambda_{h}^{1 / 2} S_{h}(t) & C_{h}(t)
\end{array}\right]
$$

with $C_{h}(t)=\cos \left(t \Lambda_{h}^{1 / 2}\right), S_{h}(t)=\sin \left(t \Lambda_{h}^{1 / 2}\right)$, which can be expressed in terms of the eigenpairs $\left\{\left(\lambda_{h, j}, \phi_{h, j}\right)\right\}_{j=1}^{N_{h}}$ of $\Lambda_{h}$.

Our weak convergence result follows.

Theorem 5.1. Let $X$ and $X_{h}$ be the solutions of (1.17) and (5.1), respectively. Let $g \in C_{\mathrm{b}}^{2}\left(\dot{H}^{0}, \mathbb{R}\right)$ and assume that $\left\|\Lambda^{\beta-\frac{1}{2}} Q \Lambda^{-\frac{1}{2}}\right\|_{\operatorname{Tr}}<\infty$ and that $X_{0} \in L_{1}\left(\Omega, H^{2 \beta}\right)$ for some $\beta \in\left[0, \frac{r+1}{2}\right]$. Then, there are $C>0, h_{0}>0$, depending on $g, X_{0}, Q$, and $T$ but not on $h$, such that for $h \leq h_{0}$,

$$
\left|\mathbf{E}\left(g\left(X_{h, 1}(T)\right)-g\left(X_{1}(T)\right)\right)\right| \leq C h^{\frac{r}{r+1} 2 \beta} .
$$

Proof. If $\left\|\Lambda^{\beta-\frac{1}{2}} Q \Lambda^{-\frac{1}{2}}\right\|_{\operatorname{Tr}}<\infty$ for some $\beta \in\left(0, \frac{r+1}{2}\right]$, then 3.5 holds by Theorem 2.1 and [10. Let $P_{1}$ denote the canonical projection $H \rightarrow H^{0}$. Define the function $G: H \rightarrow \mathbb{R}$ by $G(x):=g\left(P_{1} x\right)=g\left(x_{1}\right)$, for $x=\left[x_{1}, x_{2}\right]^{\top} \in H$. Then, by (3.8), for $y, z \in H$,

$$
\left(u_{x}(Y(t), t), y\right)=\mathbf{E}\left(\left\langle g^{\prime}\left(P_{1} Z(Y(t), t, T)\right), P_{1} y\right\rangle \mid \mathcal{F}_{t}\right)
$$

and

$$
\left(u_{x x}(Y(t), t) y, z\right)=\mathbf{E}\left(\left\langle g^{\prime \prime}\left(P_{1} Z(Y(t), t, T)\right) P_{1} y, P_{1} z\right\rangle \mid \mathcal{F}_{t}\right) .
$$

Let us introduce the error operators

$$
\begin{aligned}
& K_{h}(t):=\Lambda_{h}^{-\frac{1}{2}} S_{h}(t) P_{h}-\Lambda^{-\frac{1}{2}} S(t), \\
& G_{h}(t):=C_{h}(t) P_{h}-C(t) .
\end{aligned}
$$

From [10] we quote an error estimate for the finite element approximation of the deterministic wave equation

$$
\ddot{u}+\Lambda u=0, t>0 ; \quad u(0)=w_{1}, \dot{u}(0)=w_{2},
$$


with solution $u(t)=C(t) w_{1}+\Lambda^{-\frac{1}{2}} S(t) w_{2}$. With $w=\left[w_{1}, w_{2}\right]^{\top}$ we have

$$
\left\|G_{h}(t) w_{1}+K_{h}(t) w_{2}\right\| \leq C(T) h^{\frac{r}{r+1} s} \mid\|w\|_{s}, \quad t \in[0, T], s \in[0, r+1] .
$$

In particular, with $w_{1}=0$,

$$
\left\|K_{h}(t) w_{2}\right\| \leq C(T) h^{\frac{r}{r+1} s}\left|w_{2}\right|_{s-1}, \quad w_{2} \in \dot{H}^{s-1},
$$

or

$$
\left\|K_{h}(t) \Lambda^{\frac{1-s}{2}} v\right\| \leq C(T) h^{\frac{r}{r+1} s}\|v\|, \quad v \in \dot{H}^{1-s} .
$$

The operator $K_{h}(t) \Lambda^{\frac{1-s}{2}}$ is bounded on $\dot{H}^{0}$ for $s \geq 1$. For $0 \leq s \leq 1$ the latter estimate shows that $K_{h}(t) \Lambda^{\frac{1-s}{2}}$ extends uniquely to a bounded linear operator on $\dot{H}^{0}$ and we use the same notation for the extended operator. Hence, we may write with the operator norm and $s=2 \beta$,

$$
\left\|K_{h}(t) \Lambda^{\frac{1}{2}-\beta}\right\|_{\mathcal{B}\left(\dot{H}^{0}\right)} \leq C(T) h^{\frac{r}{r+1} 2 \beta}, \quad t \in[0, T], 0 \leq 2 \beta \leq r+1 .
$$

We use Theorem 3.1 with $G(\cdot)=g\left(P_{1} \cdot\right)$. Since $X_{h, 1}(0)=P_{h} X_{1}(0)$ and $X_{h, 2}(0)=$ $P_{h} X_{2}(0)$, we have from (5.2) and (5.4) with $s=2 \beta \leq r+1$

$$
\begin{aligned}
\mid \mathbf{E} & \left(u\left(Y_{h}(0), 0\right)-u(Y(0), 0)\right) \mid \\
& =\left|\mathbf{E}\left(\int_{0}^{1}\left(u_{x}\left(Y(0)+s\left(Y_{h}(0)-Y(0)\right), 0\right), Y_{h}(0)-Y(0)\right) \mathrm{d} s\right)\right| \\
& =\left|\int_{0}^{1} \mathbf{E}\left(\left\langle g^{\prime}\left(P_{1} Z\left(Y(0)+s\left(Y_{h}(0)-Y(0)\right)\right)\right), P_{1}\left(Y_{h}(0)-Y(0)\right)\right\rangle \mid \mathcal{F}_{0}\right) \mathrm{d} s\right| \\
& \leq \sup _{x \in \dot{H}^{0}}\left\|g^{\prime}(x)\right\| \mathbf{E}\left(\left\|P_{1}\left(Y_{h}(0)-Y(0)\right)\right\|\right) \\
& =\sup _{x \in \dot{H}^{0}}\left\|g^{\prime}(x)\right\| \mathbf{E}\left(\left\|G_{h}(T) X_{1}(0)+K_{h}(T) X_{2}(0)\right\|\right) \\
& \leq \sup _{x \in \dot{H}^{0}}\left\|g^{\prime}(x)\right\| C(T) h^{\frac{r}{r+1} 2 \beta} \mathbf{E}\left(\left.\left\|X_{0}\right\|\right|_{2 \beta}\right) .
\end{aligned}
$$

To bound the second term $(3.6)$ in the error representation in Theorem 3.1 we can simplify the integrand due to the special choice of $G$. With $y=\left[y_{1}, y_{2}\right]^{\top}$ and the abbreviation $s=T-t$ we calculate, using (5.3),

$$
\begin{aligned}
& {\left[E_{h}(s) B_{h}-E(s) B\right] Q\left[E_{h}(s) B_{h}+E(s) B\right]^{*} u_{x x}\left(Y_{h}(t), t\right)^{*} y} \\
& \quad=\mathbf{E}\left(\left[E_{h}(s) B_{h}-E(s) B\right] Q\left[E_{h}(s) B_{h}+E(s) B\right]^{*} P_{1}^{*} g^{\prime \prime}\left(P_{1} Z(Y(t), t, T)\right) P_{1} y \mid \mathcal{F}_{t}\right) .
\end{aligned}
$$

We have, using selfadjointness, that

$$
\begin{aligned}
& {\left[E_{h}(s) B_{h}+E(s) B\right]^{*} P_{1}^{*} g^{\prime \prime}\left(P_{1} Z(Y(t), t, T)\right)^{*} P_{1} y} \\
& \quad=\left(P_{1}\left[E_{h}(s) B_{h}+E(s) B\right]\right)^{*} g^{\prime \prime}\left(P_{1} Z(Y(t), t, T)\right) y_{1} \\
& \quad=\left[\Lambda_{h}^{-\frac{1}{2}} S_{h}(s) P_{h}+\Lambda^{-\frac{1}{2}} S(s)\right] g^{\prime \prime}\left(P_{1} Z(Y(t), t, T)\right) y_{1} .
\end{aligned}
$$

Therefore it follows that

$$
\begin{aligned}
& {\left[E_{h}(s) B_{h}-E(s) B\right] Q\left[E_{h}(s) B_{h}+E(s) B\right]^{*} u_{x x}\left(Y_{h}(t), t\right)^{*} y} \\
& \quad=\left[\begin{array}{l}
K_{h}(s) Q\left[\Lambda_{h}^{-\frac{1}{2}} S_{h}(s) P_{h}+\Lambda^{-\frac{1}{2}} S(s)\right] g^{\prime \prime}\left(P_{1} Z(Y(t), t, T)\right) y_{1} \\
G_{h}(s) Q\left[\Lambda_{h}^{-\frac{1}{2}} S_{h}(s) P_{h}+\Lambda^{-\frac{1}{2}} S(s)\right] g^{\prime \prime}\left(P_{1} Z(Y(t), t, T)\right) y_{1}
\end{array}\right] .
\end{aligned}
$$

Note that the above operator acts only on $y_{1}$. Therefore, when we compute its trace as in 2.4 by using an orthonormal basis for $H$ of the form $\left\{\left(e_{k}, 0\right),\left(0, f_{l}\right)\right\}_{k, l=1}^{\infty}$, 
where $\left\{e_{k}\right\}$ is an orthonormal basis of $\dot{H}^{0}$ and $\left\{f_{k}\right\}$ is an orthonormal basis of $\dot{H}^{-1}$, only terms involving $e_{k}$ remain. Hence,

$$
\begin{aligned}
\mid \mathbf{E}( & \left.\operatorname{Tr}\left(u_{x x}\left(Y_{h}(t), t\right)\left[E_{h}(s) B_{h}+E(s) B\right] Q\left[E_{h}(s) B_{h}-E(s) B\right]^{*}\right)\right) \mid \\
= & \left|\mathbf{E}\left(\operatorname{Tr}\left(\left[E_{h}(s) B_{h}-E(s) B\right] Q\left[E_{h}(s) B_{h}+E(s) B\right]^{*} u_{x x}\left(Y_{h}(t), t\right)^{*}\right)\right)\right| \\
= & \left|\mathbf{E}\left(\sum_{k=1}^{\infty} \mathbf{E}\left(\left\langle K_{h}(s) Q\left[\Lambda_{h}^{-\frac{1}{2}} S_{h}(s) P_{h}+\Lambda^{-\frac{1}{2}} S(s)\right] g^{\prime \prime}\left(P_{1} Z(Y(t), t, T)\right) e_{k}, e_{k}\right\rangle \mid \mathcal{F}_{t}\right)\right)\right| \\
= & \left|\mathbf{E}\left(\operatorname{Tr}\left(K_{h}(s) Q\left[\Lambda_{h}^{-\frac{1}{2}} S_{h}(s) P_{h}+\Lambda^{-\frac{1}{2}} S(s)\right] g^{\prime \prime}\left(P_{1} Z(Y(t), t, T)\right)\right)\right)\right| \\
\leq & \left\|K_{h}(s) \Lambda^{\frac{1}{2}-\beta}\right\|_{\mathcal{B}\left(\dot{H}^{0}\right)}\left\|\Lambda^{\beta-\frac{1}{2}} Q \Lambda^{-\frac{1}{2}}\right\|_{\operatorname{Tr}} \\
& \times\left\|\Lambda^{\frac{1}{2}}\left[\Lambda_{h}^{-\frac{1}{2}} S_{h}(s) P_{h}+\Lambda^{-\frac{1}{2}} S(s)\right]\right\|_{\mathcal{B}\left(\dot{H}^{0}\right)} \sup _{x \in \dot{H}^{0}}\left\|g^{\prime \prime}(x)\right\|_{\mathcal{B}\left(\dot{H}^{0}\right)} .
\end{aligned}
$$

Noting that $\left\|\Lambda^{\frac{1}{2}} v_{h}\right\|=\left\|\nabla v_{h}\right\|=\left\|\Lambda_{h}^{\frac{1}{2}} v_{h}\right\|$ for $v_{h} \in S_{h}$, and using (5.5), we conclude

$$
\begin{aligned}
& \left|\mathbf{E}\left(\operatorname{Tr}\left(u_{x x}\left(Y_{h}(t), t\right)\left[E_{h}(s) B_{h}+E(s) B\right] Q\left[E_{h}(s) B_{h}-E(s) B\right]^{*}\right)\right)\right| \\
& \quad \leq C(T) h^{\frac{r}{r+1} 2 \beta}\left\|\Lambda^{\beta-\frac{1}{2}} Q \Lambda^{-\frac{1}{2}}\right\|_{\operatorname{Tr}} \sup _{x \in \dot{H}^{0}}\left\|g^{\prime \prime}(x)\right\|_{\mathcal{B}\left(\dot{H}^{0}\right)} .
\end{aligned}
$$

Now, we may estimate the second term (3.6),

$$
\begin{aligned}
& \mid \mathbf{E}\left(\int _ { 0 } ^ { T } \operatorname { T r } \left(u_{x x}\left(Y_{h}(t), t\right)\right.\right. \\
& \left.\left.\quad \times\left[E_{h}(T-t) B_{h}+E(T-t) B\right] Q\left[E_{h}(T-t) B_{h}-E(T-t) B\right]^{*}\right) \mathrm{~d} t\right) \mid \\
& \quad \leq C(T) h^{\frac{r}{r+1} 2 \beta}\left\|\Lambda^{\beta-\frac{1}{2}} Q \Lambda^{-\frac{1}{2}}\right\|_{\operatorname{Tr}} \sup _{x \in \dot{H}^{0}}\left\|g^{\prime \prime}(x)\right\|_{\mathcal{B}\left(\dot{H}^{0}\right)} .
\end{aligned}
$$

In 10] the strong rate of convergence is found to be $O\left(h^{\frac{r}{r+1}} \beta\right)$ for $\beta \in[0, r+1]$ under the condition $\left\|\Lambda^{\frac{\beta-1}{2}} Q^{\frac{1}{2}}\right\|_{\mathrm{HS}}<\infty$. Theorem 2.1 shows that $\left\|\Lambda^{\beta-\frac{1}{2}} Q \Lambda^{-\frac{1}{2}}\right\|_{\operatorname{Tr}}<$ $\infty$ provides a sufficient condition for this and the conditions coincide if $A$ and $Q$ commute, in particular, if $Q=I$.

As a special case, if $Q=I$, then $d=1$ and we may take $\beta<\frac{1}{2}$. Hence the order of weak convergence is almost $O\left(h^{\frac{r}{r+1}}\right)$.

\section{REFERENCES}

[1] G. Da Prato and J. Zabczyk, Stochastic Equations in Infinite Dimensions, Cambridge University Press, Cambridge, 1992.

[2] __ Second Order Partial Differential Equations in Hilbert Spaces, Cambridge University Press, Cambridge, 2002.

[3] A. de Bouard and A. Debussche, Weak and strong order of convergence of a semidiscrete scheme for the stochastic nonlinear Schrödinger equation, Appl. Math. Optim. 54 (2006), 369-399.

[4] A. Debussche, Weak approximation of stochastic partial differential equations: the non linear case, preprint 2008, arXiv:0804.1304v1 [math.NA].

[5] A. Debussche and J. Printems, Weak order for the discretization of the stochastic heat equation, Math. Comp. 78 (2009), 845-863.

[6] C. M. Elliott and S. Larsson, Error estimates with smooth and nonsmooth data for the finite element method for the Cahn-Hilliard equation, Math. Comp. 58 (1992), 603-630. 
[7] E. Hausenblas, Weak approximation for semilinear stochastic evolution equations, Stochastic Analysis and Related Topics VIII, Progr. Probab., vol. 53, Birkhäuser, Basel, 2003, pp. 111128.

[8] E. Hausenblas, Weak approximation of the stochastic wave equation, preprint.

[9] M. Geissert, M. Kovács and S. Larsson, Rate of weak convergence of the finite element method for the stochastic heat equation with additive noise, BIT 49 (2009), 343-356.

[10] M. Kovács, S. Larsson and F. Saedpanah, Finite element approximation of the linear stochastic wave equation with additive noise, SIAM J. Numer. Anal. 48 (2010), 408-427.

[11] S. Larsson and A. Mesforush, Finite element approximation of the linearized Cahn-HilliardCook equation, IMA J. Numer. Anal, to appear.

[12] P. D. Lax, Functional Analysis, Wiley, 2002.

[13] V. Thomée, Galerkin Finite Element Methods for Parabolic Problems, 2nd ed., Springer, 2006.

[14] Y. Yan, Semidiscrete Galerkin approximation for a linear stochastic parabolic partial differential equation driven by an additive noise, BIT 44 (2004), 829-847.

[15] J. Weidmann, Linear Operators in Hilbert Spaces, Springer, 1980.

Department of Mathematics and Statistics, University of Otago, P.O. Box 56, DuneDin, New Zealand

E-mail address: mkovacs@maths.otago.ac.nz

Department of Mathematical Sciences, Chalmers University of Technology and UniVersity of Gothenburg, SE-412 96 Göteborg, SWEden

E-mail address: stig@chalmers.se

Department of Mathematical Sciences, Chalmers University of Technology and University of Gothenburg, SE-41296 Göteborg, Sweden

E-mail address: fredrik.lindgren@chalmers.se 